Article

\title{
TmSpz6 Is Essential for Regulating the Immune Response to Escherichia coli and Staphylococcus aureus Infection in Tenebrio molitor
}

\author{
Tariku Tesfaye Edosa ${ }^{1,2,+} \mathbb{D}$, Yong Hun Jo ${ }^{1,+} \mathbb{C}$, Maryam Keshavarz ${ }^{1} \mathbb{D}$, Young Min Bae ${ }^{1}$, \\ Dong Hyun Kim ${ }^{1}$, Yong Seok Lee ${ }^{3}$ (i) and Yeon Soo Han ${ }^{1, *(D)}$ \\ 1 Department of Applied Biology, Institute of Environmentally-Friendly Agriculture (IEFA), \\ College of Agriculture and Life Sciences, Chonnam National University, Gwangju 61186, Korea; \\ bunchk.2000@gmail.com (T.T.E.); yhun1228@jnu.ac.kr (Y.H.J.); mariakeshavarz1990@gmail.com (M.K.); \\ ugisaka@naver.com (Y.M.B.); kjsdh3@hotmail.com (D.H.K.) \\ 2 Ethiopian Institute of Agricultural Research, Ambo Agricultural Research Center, Ambo 37, Ethiopia \\ 3 Department of Life Science and Biotechnology, College of Natural Sciences, Soonchunhyang University, \\ Asan 31538, Korea; yslee@sch.ac.kr \\ * Correspondence: hanys@jnu.ac.kr; Tel.: +82-62-530-2072 \\ + These authors have equally contributed in this study.
}

Received: 14 January 2020; Accepted: 3 February 2020; Published: 5 February 2020

check for updates

\begin{abstract}
Spätzle is an extracellular protein that activates the Toll receptor during embryogenesis and immune responses in Drosophila. However, the functions of the spätzle proteins in the innate immune response against bacteria or fungi in T. molitor are not well understood. Therefore, in this study, the open reading frame (ORF) of TmSpz6 was identified and its function in the response to bacterial and fungal infections in T. molitor was investigated using RNAi. The highest expression of TmSpz6 was in prepupae, and 3- and 6-day-old pupae, while remarkable expression was also observed in other stages. The tissue-specific expression analysis showed that TmSpz6 expression was highest in the hemocytes of larvae. TmSpz6 expression was highly induced when challenged with Escherichia coli, Staphylococcus aureus, or Candida albicans at $6 \mathrm{~h}$ post-injection; however, TmSpz6-silenced larvae were significantly more susceptible to only E. coli and S. aureus infection. The antimicrobial peptides (AMPs) gene expression analysis results show that TmSpz6 mainly positively regulated the expression of TmTencin-2 and -3 in response to E. coli and S. aureus infection. Collectively, these results suggest that TmSpz6 plays an important role in regulating AMP expression and increases the survival of $T$. molitor against E. coli and S. aureus.
\end{abstract}

Keywords: antimicrobial peptides; immune response; mealworm; spätzle; Toll pathway

\section{Introduction}

Insects have well-developed and complex innate immune systems that are divided into humoral and cellular components. The humoral immune system principally consists of antimicrobial peptides (AMPs), lectins, lysozyme, protease inhibitors, and other factors [1]. The recognition of invading pathogens leads to the activation of signal transduction pathways, such as Toll or immune deficiency (IMD), and ultimately induces the expression of antimicrobial peptides (AMPs) that attack the invading pathogens $[2,3]$.

In Drosophila, AMP production is controlled by downstream signal transduction pathway that includes spätzle, Toll, tube, pelle, and cactus [4-7]. This pathway is activated when pattern-recognition receptors (PRRs) recognize the pathogen-associated molecular pattern [8] and convey the signal to a 
proteolytic cascade that leads to the cleavage of the cytokine-like protein pro-spätzle; the resulting cleaved, mature spätzle activates the Toll receptor as a ligand $[9,10]$.

Binding of spätzle to the Toll receptor activates a downstream signaling pathway containing several protein complexes, including adaptor proteins such as myeloid differentiation primary response 88 (MyD88), Tube, and the serine-threonine innate immunity kinase (Pelle) [11]. Signaling through the MyD88-Tube-Pelle complex leads to the phosphorylation and degradation of the I $\mathrm{KB}$ factor Cactus, which inhibits the nuclear localization and activity of NF- $\mathrm{kB}$ transcription factors. Once Cactus is degraded, both Dorsal and Dif translocate to the nucleus and bind to $\mathrm{kB}$-related sequences in the AMP genes [12].

Initially, spätzle exists as an inactive extracellular pro-spätzle, which contains a signal peptide, an N-terminal regulatory cleavage site, and a signaling precursor consisting of a 106-amino acid C-terminal active fragment $[13,14]$. The active ligand spätzle is generated during development or immune response [14].

In Tenebrio molitor, upon infection, the recognition of Lys-type peptidoglycan (PG) (gram-positive bacteria) or $\beta$-1,3-glucan (fungi) and DAP-type PG (gram-negative bacteria) by PGRP-SA/GNBP1 or PGRP-LE/LC activates a serine protease cascade. This cascade, which includes modular serine protease (MSP), spätzle processing enzyme activating enzyme (SAE), and spätzle-processing enzyme (SPE), mediates the cleavage of pro-spätzle and the generation of active spätzle $[9,15]$. The importance of spätzle in the Toll pathway has been demonstrated in insects such as Drosophila melanogaster [16, 17], Bombyx mori [18], Manduca sexta [19], and mosquitoes [20,21], as well as shrimps, including Fenneropenaeus chinensis [22] and Litopenaeus vannamei [23].

Spätzle proteins have been shown to be important in the innate immune response to microbial infection in various arthropods from insects to shrimp. However, apart from a biochemical characterization [9], the functions of the spätzle genes in the T. molitor immune system in response to microbial challenge have not been yet studied. Therefore, the current study focused on the identification and functional characterization of TmSpätzle 6 in innate immune response to various pathogens in T. molitor.

\section{Materials and Methods}

\subsection{Insect Culture}

T. molitor (mealworm) was maintained at $27 \pm 1{ }^{\circ} \mathrm{C}$ and $60 \pm 5 \%$ relative humidity in the dark on an artificial diet containing $170 \mathrm{~g}$ of whole-wheat flour, $20 \mathrm{~g}$ of fried bean powder, $10 \mathrm{~g}$ of soy protein, $100 \mathrm{~g}$ of wheat bran, $200 \mathrm{~mL}$ of sterile water, $0.5 \mathrm{~g}$ of chloramphenicol, $0.5 \mathrm{~g}$ of sorbic acid, and $0.5 \mathrm{~mL}$ of propionic acid. For the experiments, 10th-12th instar larvae were used. To ensure that uniformly sized larvae were used, the larvae were separated according to size using a set of laboratory test sieves (Pascall Eng. Co., Ltd., Crawley, Sussex, England).

\subsection{Preparation of Microorganisms}

The gram-negative bacterium Escherichia coli K12, the gram-positive bacterium Staphylococcus aureus RN4220, and the fungus Candida albicans were used to study the function of TmSpz6 in the innate immune response of mealworms against microbial infection. These microorganisms have been repeatedly used by various researchers to study the immune related gene expression [24-26]. The microorganisms were cultured in Luria-Bertani (LB; E. coli and S. aureus) and Sabouraud dextrose (C. albicans) broths at $37^{\circ} \mathrm{C}$ overnight and were subsequently subcultured at $37^{\circ} \mathrm{C}$ for $3 \mathrm{~h}$. Then, the microorganisms were harvested and washed two times by centrifugation at $3500 \mathrm{rpm}$ for $10 \mathrm{~min}$ in phosphate-buffered saline (PBS; pH 7.0). The washed microorganisms were suspended in PBS and their optical densities were measured at $600 \mathrm{~nm}\left(\mathrm{OD}_{600}\right)$ to determine the concentration. Finally, $10^{6}$ cells $/ \mu \mathrm{L}$ of $E$. coli or $S$. aureus and $5 \times 10^{4}$ cells $/ \mu \mathrm{L}$ of $C$. albicans were separately injected into the larvae. 


\subsection{Identification and Cloning of the Full-length TmSpz6 cDNA}

The T. molitor TmSpz6 gene was identified by a local-tBLASTn analysis using the amino acid sequence of the T. castaneum spz6 gene (NP_001164082.1) as the query. The partial cDNA sequence of Tmspz 6 was obtained from the T. molitor RNAseq database, and the full-length cDNA sequence of TmSpz 6 was identified by the $5^{\prime}$ - and 3'-rapid amplification of cDNA end (RACE) polymerase chain reaction (PCR) using a SMARTer RACE cDNA amplification kit (Clontech, Mountain View, CA, USA) according to the manufacturer's instructions. The PCR was performed with AccuPower ${ }^{\circledR}$ PyroHotStart Taq PCR PreMix (Bioneer, Korea) and TmSpz6-specific primers (TmSpz6-cloning_Fw and TmSpz6-cloning_Rv; Table 1) under the following cycling conditions: a pre-denaturation step at $95^{\circ} \mathrm{C}$ for $5 \mathrm{~min}$, followed by 35 cycles of denaturation at $95^{\circ} \mathrm{C}$ for $30 \mathrm{~s}$, annealing at $53^{\circ} \mathrm{C}$ for $30 \mathrm{~s}$, and extension at $72{ }^{\circ} \mathrm{C}$ for $2 \mathrm{~min}$, with a final extension step at $72{ }^{\circ} \mathrm{C}$ for $5 \mathrm{~min}$ on a MyGenie96 Thermal Block (Bioneer, Korea). The PCR products were purified using the AccuPrep ${ }^{\circledR}$ PCR Purification Kit (Bioneer, Korea), immediately ligated into T-Blunt vectors (Solgent, Korea), and transformed into $E$. coli DH5 $\alpha$ competent cells according to the manufacturer's instructions. Plasmid DNA was extracted from the transformed cells using the AccuPrep ${ }^{\circledR}$ Nano-Plus Plasmid Extraction Kit (Bioneer, Korea), sequenced, and analyzed. Finally, the full-length cDNA sequence of TmSpz 6 was obtained.

\subsection{Domain and Phylogenetic Analyses}

Specific domains were analyzed using the InterProScan 5 and BLASTp programs. A multiple sequence alignment was performed with representative Spz6 protein sequences from other insects obtained from GenBank using ClustalX2 software. Phylogenetic and percentage identity analyses were conducted using ClustalX2 and MEGA 7 programs.

\subsection{Analysis of TmSpz6 Expression and Induction Patterns}

To study the expression of TmSpz6 across development, total RNA was extracted from whole T. molitor $(\mathrm{n}=20)$ eggs $(\mathrm{EG})$, young instar larvae (YL; 10th-12th instar larvae), late instar larvae (LL; 19th-20th instar larvae), prepupae (PP), 1-7-day-old pupae (P1-P7), and 1-5-day-old adults (A1-A5). To investigate the tissue-specific expression patterns of TmSpz6, RNA was extracted from the gut, hemocytes, integument, Malpighian tubules, and fat body of late instar larvae and adults as well as the ovaries and testes of adults $(\mathrm{n}=20)$. The TmSpz6 gene expression induction pattern in T. molitor larval tissues was analyzed following injection with E. coli, S. aureus, or C. albicans into young instar larvae. Three immune response-related tissues, the hemocytes, fat body, and gut, were collected at 3, 6, 9, 12, and $24 \mathrm{~h}$ post-injection.

The samples were collected into $500 \mu \mathrm{L}$ of guanidine thiocyanate RNA lysis buffer $(2 \mathrm{~mL}$ of 0.5 M EDTA, $1 \mathrm{~mL}$ of $1 \mathrm{M}$ MES Buffer, $17.72 \mathrm{~g}$ of guanidine thiocyanate, $0.58 \mathrm{~g}$ of sodium chloride, 0.7 mg of phenol red, $25 \mu \mathrm{L}$ of Tween-80, $250 \mu \mathrm{L}$ of glacial acetic acid, and $500 \mu \mathrm{L}$ of isoamyl alcohol) and were homogenized with a homogenizer (Bertin Technologies, France) at $7500 \mathrm{rpm}$ for $20 \mathrm{~s}$. The total RNA was extracted using the modified LogSpin RNA isolation method [27], and $2 \mu \mathrm{g}$ of the extracted total RNA was used to immediately synthesize cDNA with AccuPower ${ }^{\circledR}$ RT PreMix (Bioneer, Korea) and Oligo (dT) 12-18 primers on a MyGenie96 Thermal Block (Bioneer, Korea) according to the manufacturer's instructions.

Real-Time Quantitative PCR (RT-qPCR) was performed on an Exicycler ${ }^{\mathrm{TM}} 96$ Real-Time Quantitative Thermal Block (Bioneer, Daejeon, Korea) with gene-specific primers under the following cycling conditions: an initial denaturation step at $94^{\circ} \mathrm{C}$ for $5 \mathrm{~min}$, followed by 45 cycles of denaturation at $95^{\circ} \mathrm{C}$ for $15 \mathrm{~s}$ and annealing at $60^{\circ} \mathrm{C}$ for $30 \mathrm{~s}$. The $2^{-\Delta \Delta \mathrm{Ct}}$ method [28] was used to analyze $\mathrm{TmSpz} 6$ expression levels. T. molitor ribosomal protein L27a (TmL27a) was used as an internal control to normalize differences in template concentration between samples. 
Table 1. Primers used in this study.

\begin{tabular}{|c|c|}
\hline Primer Name & Sequence $\left(5^{\prime}-3^{\prime}\right)$ \\
\hline TmSpz6-qPCR-Fw & GCACAACTCCAAGACGACCT \\
\hline TmSpz6-qPCR-Rv & TCTCTTCACCCGATCGTTGC \\
\hline TmSpz6-T7-Fw & TAATACGACTCACTATAGGGTACCGCGCAAGAGAGTAAAAA \\
\hline TmSpz6-T7-Rv & TAATACGACTCACTATAGGGTACGTATCTCCACACCCCTTG \\
\hline TmSpz6-cloning-Fw & CCCCTGTCGACTGCAAAGAA \\
\hline TmSpz6-cloning-Rv & CACCACGTATCTCCACACCC \\
\hline TmSpz6-cloning-FullORF-Fw & TGAGTGAATAATTTCGAAAAGAAAAA \\
\hline TmSpz6-cloning-FullORF-Rv & TGGGCGTTCAGTTACATCAA \\
\hline TmL27a_qPCR_Fw & TCATCCTGAAGGCAAAGCTCCAGT-3' \\
\hline TmL27a_qPCR_Rv & AGGTTGGTTAGGCAGGCACCTTTA-3' \\
\hline dsEGFP_Fw & TAATACGACTCACTATAGGGTCGTAAACGGCCACAAGTTC \\
\hline dsEGFP_Rv & TAATACGACTCACTATAGGGTTGCTCAGGTAGTGTTGTCG \\
\hline TmTencin-1_Fw & CAGCTGAAGAAATCGAACAAGG \\
\hline TmTencin-1_Rv & CAGACCCTCTTTCCGTTACAGT \\
\hline TmTencin-2_Fw & CAGCAAAACGGAGGATGGTC \\
\hline TmTencin-2_Rv & CGTTGAAATCGTGATCTTGTCC \\
\hline TmTencin-3_Fw & GATTTGCTTGATTCTGGTGGTC \\
\hline TmTencin-3_Rv & CTGATGGCCTCCTAAATGTCC \\
\hline TmTencin-4_Fw & GGACATTGAAGATCCAGGAAAG \\
\hline TmTencin-4_Rv & CGGTGTTCCTTATGTAGAGCTG \\
\hline TmDefensin-1_Fw & AAATCGAACAAGGCCAACAC \\
\hline TmDefencin-1_Rv & GCAAATGCAGACCCTCTTTC \\
\hline TmDefencin-2_Fw & GGGATGCCTCATGAAGATGTAG \\
\hline TmDefencin-2_Rv & CCAATGCAAACACATTCGTC \\
\hline TmColoptericin-1_Fw & GGACAGAATGGTGGATGGTC \\
\hline TmColoptericin-1_Rv & CTCCAACATTCCAGGTAGGC-3 \\
\hline TmColoptericin-2_Fw & GGACGGTTCTGATCTTCTTGAT \\
\hline TmColoptericin-2_Rv & CAGCTGTTTGTTTGTTCTCGTC \\
\hline TmAttacin-1a_Fw & GAAACGAAATGGAAGGTGGA \\
\hline TmAttacin-1a_Rv & TGCTTCGGCAGACAATACAG \\
\hline TmAttacin-1b_Fw & GAGCTGTGAATGCAGGACAA \\
\hline TmAttacin-1b_Rv & CССТCTGATGAAACCTCCAA \\
\hline TmAttacin-2_Fw & AACTGGGATATTCGCACGTC \\
\hline TmAttacin-2_Rv & CCCTCCGAAATGTCTGTTGT-3 \\
\hline TmCecropin-2_Fw & TACTAGCAGCGCCAAAACCT \\
\hline TmCecropin-2_Rv & CTGGAACATTAGGCGGAGAA \\
\hline TmThaumatin-like protein-1_Fw & CTCAAAGGACACGCAGGACT \\
\hline TmThaumatin-like protein-1_Rv & ACTTTGAGCTTCTCGGGACA \\
\hline TmThaumatin-like protein-2_Fw & CCGTCTGGCTAGGAGTTCTG \\
\hline TmThaumatin-like protein-2_Rv & ACTCCTCCAGCTCCGTTACA \\
\hline cates $\mathrm{T} 7$ promoter sequ & \\
\hline
\end{tabular}

\subsection{RNA Interference Analysis}

To synthesize the double-stranded (ds) RNA of the TmSpz6 gene, primers containing the T7 promoter sequence at their $5^{\prime}$ ends were designed using SnapDragon-Long dsRNA design software (Table 1). The PCR was conducted using AccuPower ${ }^{\circledR}$ Pfu PCR PreMix with the TmSpz6_Fw and TmSpz6_Rv primers (Table 1) under the following cycling conditions: an initial denaturation step at $94{ }^{\circ} \mathrm{C}$ for $2 \mathrm{~min}$ followed by 35 cycles of denaturation at $94{ }^{\circ} \mathrm{C}$ for $30 \mathrm{~s}$, annealing at $53^{\circ} \mathrm{C}$ for $30 \mathrm{~s}$, and extension at $72{ }^{\circ} \mathrm{C}$ for $30 \mathrm{~s}$, with a final extension step at $72{ }^{\circ} \mathrm{C}$ for $5 \mathrm{~min}$. PCR products were purified using the AccuPrep PCR Purification Kit (Bioneer, Daejeon, Korea), and dsRNA was synthesized using the Ampliscribe ${ }^{\mathrm{TM}}$ T7-Flash ${ }^{\mathrm{TM}}$ Transcription Kit (Epicentre Biotechnologies, Madison, WI, USA) according to the manufacturer's instructions. After synthesis, the dsRNA was purified by precipitation with $5 \mathrm{M}$ ammonium acetate and $80 \%$ ethanol, and then it was quantified with an Epoch spectrophotometer (BioTek Instruments, Inc., Winooski, VT, USA). The dsRNA for enhanced green fluorescent protein (dsEGFP) was synthesized for use as a control and was stored at $-20^{\circ} \mathrm{C}$ until use. 


\subsection{Effect of TmSpz6 Gene Silencing on the Response to Microorganism Challenge}

To study the importance of TmSpz6 in the T. molitor immune response, dsTmSpz6 $(1 \mu \mathrm{g} / \mu \mathrm{L})$ was first injected into young-instar larvae (10th-12th instars; $n=30)$ using disposable needles mounted onto a micro-applicator (Picospiritzer III Micro Dispense System; Parker Hannifin, Hollis, NH, USA). An equal amount of dsEGFP was injected in similar larvae as a negative control. TmSpz6 knockdown was evaluated, and over 90\% knockdown was achieved at 2 days post-injection. Second, the TmSpz6-silenced and dsEGFP-injected larval groups were challenged with E. coli $\left(10^{6}\right.$ cells $\left./ \mu \mathrm{L}\right), S$. aureus $\left(10^{6}\right.$ cells $\left./ \mu \mathrm{L}\right)$, or $C$. albicans $\left(5 \times 10^{4}\right.$ cells $\left./ \mu \mathrm{L}\right)$ in triplicate experiments. The challenged larvae were maintained for 10 days, during which the number of surviving larvae were recorded. The survival rates of the TmSpz6-silenced larvae were compared to survival of control larvae.

\subsection{Effect of dsTmSpz6 on AMP Expression in Response to Microbial Challenge}

To characterize the function of TmSpz6 in regulating AMP gene expression in response to microbial infection, TmSpz6 gene expression in larvae was silenced with RNAi and then these larvae were injected with microorganisms (E. coli, S. aureus, or C. albicans). dsEGFP and PBS were used as the negative and injection controls, respectively. At $24 \mathrm{~h}$ post-injection, the hemocytes, fat body, and gut were dissected, and then total RNA was extracted from these tissues and cDNA was synthesized as described above. Next, qRT-PCR was conducted using specific primers (Table 1) to analyze the temporal expression patterns of 14 AMP genes: TmTencin-1 (TmTen-1), TmTencin-2 (TmTen2), TmTencin-3 (TmTen-3), TmTencin-4 (TmTen-4), TmAttacin-1a (TmAtt-1a), TmAttacin-1b (TmAtt-1b), TmAttacin-2 (TmAtt-2), TmDefensin-1 (TmDef-1), TmDefensin-2 (TmDef-2), TmColeoptericin-1 (TmCol-1), TmColeoptericin-2 (TmCol-2), TmCecropin-2 (TmCec-2), TmThaumatin like protein-1 (TmTLP-1), and TmThaumatin like protein-2 (TmTLP-2).

\subsection{Data Analysis}

Statistical analyses were conducted using SAS 9.4 software (SAS Institute, Inc., Cary, NC, USA), and cumulative survival was analyzed by Tukey's multiple test, at a significance level of $p<0.05$. AMP gene expression was calculated using the delta $\mathrm{Ct}(\Delta \Delta \mathrm{Ct})$ method, and the fold change compared to the internal control (TmL27a) and external control (PBS) was calculated by the $2^{(\Delta \Delta \mathrm{Ct})}$ method.

\section{Results}

\subsection{Sequence Identification and Phylogenetic Analysis of TmSpz6}

The full-length open reading frame (ORF) sequence of TmSpz6 was obtained from the T. molitor RNAseq database and by 5' - and 3'-RACE PCR. The TmSp6 ORF is 1227 bp and encodes 408 amino acid residues (Figure 1). Domain analysis indicated that TmSpz6 contains one cystine-knot domain at the C-terminus, one cleavage site, and one signal peptide region. Phylogenetic analysis revealed that TmSpz6 grouped together with another spätzle 6 from a Coleopteran insect (Tribolium castaneum spätzle 6) (Figure 2).

\subsection{Developmental and Tissue-Specific Expression Patterns of TmSpz6}

The developmental and tissue-specific expression patterns of TmSpz 6 mRNA were examined by RT-qPCR. TmSpz6 transcripts were observed in all tested developmental stages and tissues (Figure 3). TmSpz 6 was expressed at similar levels in young and late larvae, but was highly expressed in pre-pupae. Expression was decreased in 1- and 2-day-old pupae but was then highly upregulated and peaked in 3-day-old pupae. This was followed by a gradual decrease in expression in 4- and 5-day-old pupae and an increase in 6-day-old pupae. During the adult stage, TmSpz6 was expressed at similar levels at all examined ages, except it was slightly lower in 5-day-old adults (Figure 3A). 


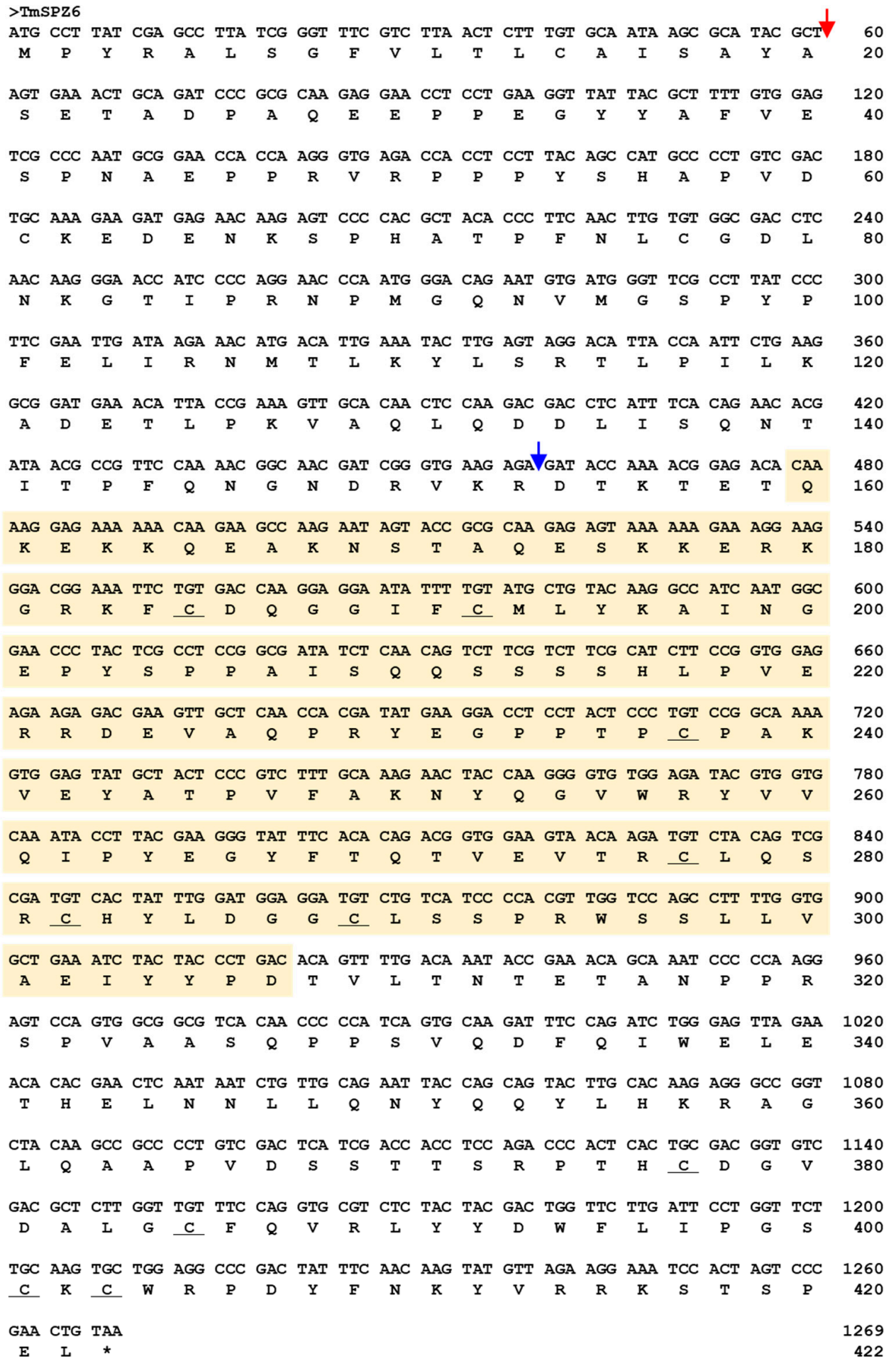

Figure 1. Nucleotide and deduced amino acid sequences of TmSpz6. It consists of a 1227 bp open reading frame encoding a 408 amino acid residue protein. Domain analysis showed that TmSpz6 includes one cystine-knot domain (yellow box), one signal peptide (red arrow), and one cleavage site (blue arrow). 


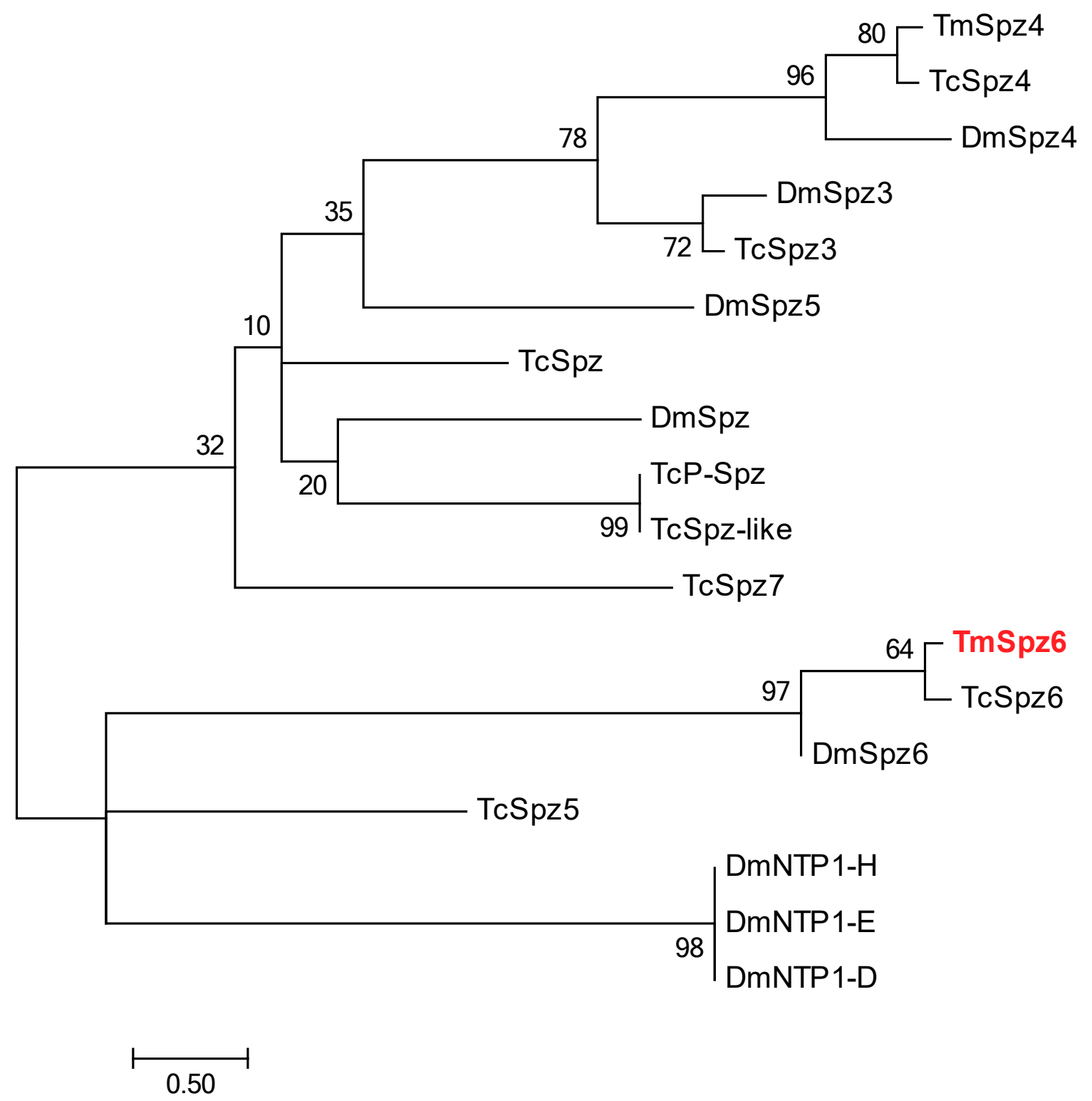

Figure 2. Molecular phylogenetic analysis of insect Spz6 homologs. Phylogenetic analyses of TmSpz6 homologues were performed using ClustalX2, and the phylogenic tree was constructed with MEGA7 using the maximum likelihood method and 1000 bootstrapped replications. The following protein sequences were used to construct the phylogenetic tree. DmSpz4, Drosophila melanogaster spätzle 4 (AAF53100.2); DmSpz6, D. melanogaster spätzle 6 (AAF47261.1); DmSpz5, D. melanogaster spätzle 5 (AAF47694.1); DmSpz, D. melanogaster spätzle (AAF82745.1); DmSpz3, D. melanogaster spätzle 3 (AAF52574.2); DmNTP1-H, D. melanogaster neurotrophin 1, isoform H (AGB94113.1); DmNTP1-E, D. melanogaster neurotrophin 1, isoform E (ACZ94621.1); DmNTP1-D, D. melanogaster neurotrophin 1, isoform D (NP_001163348.1); TcSpz7, Tribolium castaneum spätzle 7 (EEZ99267.2); TcSpz4, T. castaneum spätzle 4 (EFA09263.2); TcSpz5, T. castaneum spätzle 5 (EEZ97725.1); TcSpz3, T. castaneum spätzle 3 (NP_001153625.1); TcSpz6, T. castaneum spätzle 6 precursor (NP_001164082.1); TcSpz, T. castaneum spätzle (EEZ99207.1); TcP-Spz, T. castaneum PREDICTED protein spätzle (XP_008201191.1); TcSpz-like, T. castaneum spätzle-like protein (EEZ99268.2). 

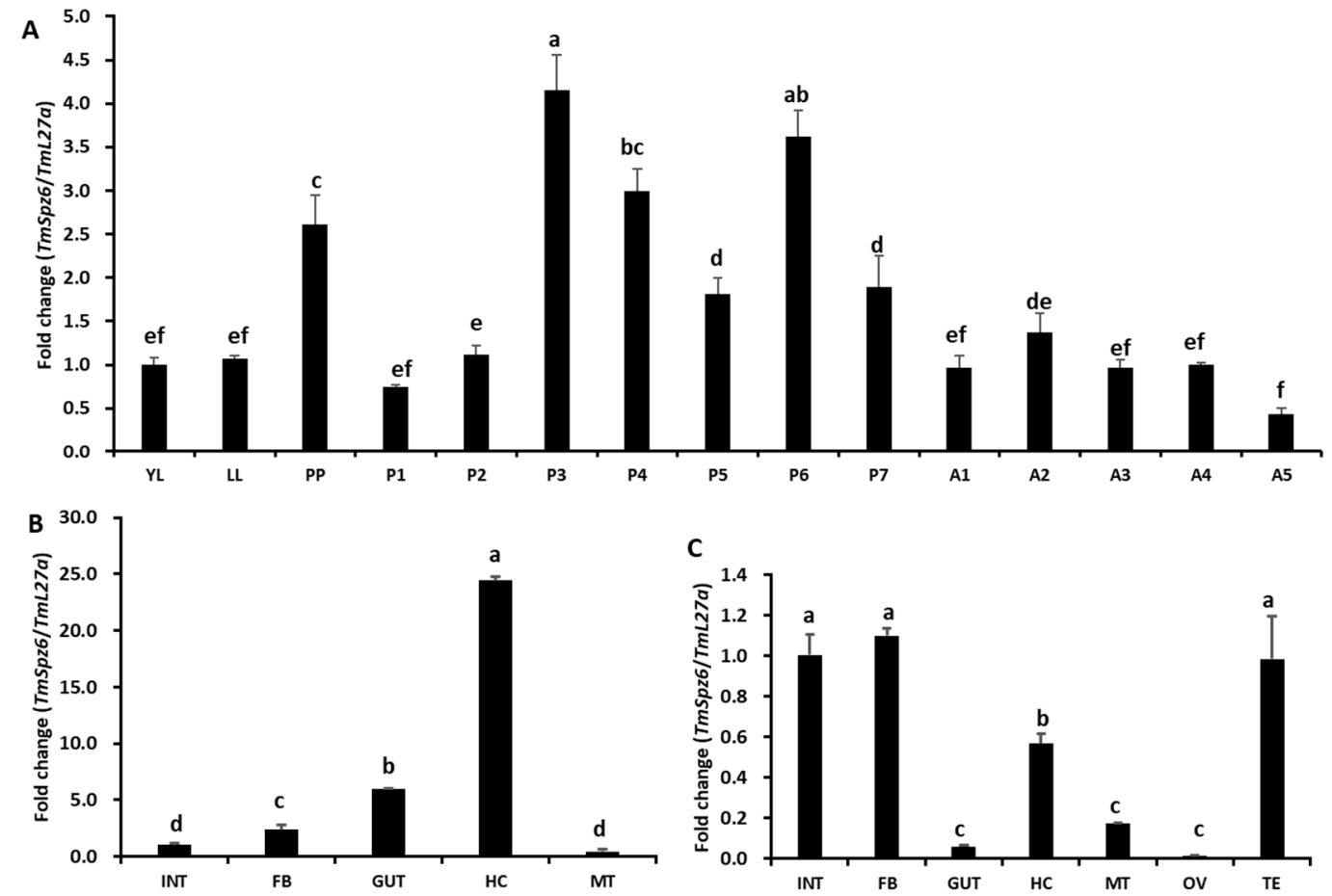

Figure 3. Developmental stage- and tissue-specific expression patterns of the TmSpz6 gene in T. molitor. TmSpz6 expression patterns across the developmental stages of T. molitor, including, young larvae (YL), late larvae (LL), pre-pupa (PP), 1-7-day-old pupae (P1-7), and 1-5-day-old adults (A1-5) were examined (A). For each sample, RNA extracted from 20 individuals was used to synthesis cDNA. The results indicate that TmSpz 6 expression gradually increased from the young larval to pre-pupal stage. In the pupal stage, the highest expression was observed in 4-day-old pupae. In adults, there was no considerable difference in expression in 2-5-day-old adults. The tissue-specific expression patterns of TmSpz6 were also assessed in late larvae (B) and 5-day-old adults (C). The hemocytes (HC), gut, fat body (FB), Malpighian tubules (MT), and integument (INT) (for late instar larvae and adults), as well as the testes (TE) and ovaries (OV) (for adults), were dissected and collected from 20 late larvae and 5-day-old adults. T. molitor $60 \mathrm{~S}$ ribosomal protein L27a (TmL27a) served as an endogenous control to normalize RNA levels between samples. The data are the means of three biological replicates. The asterisks indicate significant differences $(p \leq 0.05)$.

The examination of different tissues revealed that TmSpz6 was highly expressed in hemocytes, but was expressed at lower levels in the integument, fat body, and Malpighian tubules of late instar larvae (Figure 3B). In adults, expression was high in the integument and fat body followed by the testis and hemocytes (Figure 3C). Comparatively low TmSpz6 expression levels were detected in the gut, Malpighian tubules, and ovaries of 5-day-old adults.

\subsection{Temporal Induction of TmSpz6 after Microbial Challenge}

To determine the inducibility of TmSpz6 during microbial infection, the temporal expression of TmSpz6 in T. molitor larvae was examined after injecting E. coli, S. aureus, or C. albicans. The temporal expression patterns in three immune-related tissues, hemocytes, fat body, and gut, were analyzed at $3,6,9,12$, and $24 \mathrm{~h}$ post-injection by RT-qPCR. The microbial challenge time-dependently induced transcription of TmSpz 6 in all tested tissues. All injected microorganisms induced the highest TmSpz6 expression in hemocytes at 6-h post injection (Figure 4A). In E. coli- and S. aureus-injected larvae, the highest TmSpz6 expression in the gut was observed at $9 \mathrm{~h}$ post injection (Figure 4B), whereas in E. coliand C. albicans-injected larvae, the highest expression in the fat body was detected at $24 \mathrm{~h}$ post-injection (Figure 4C). 

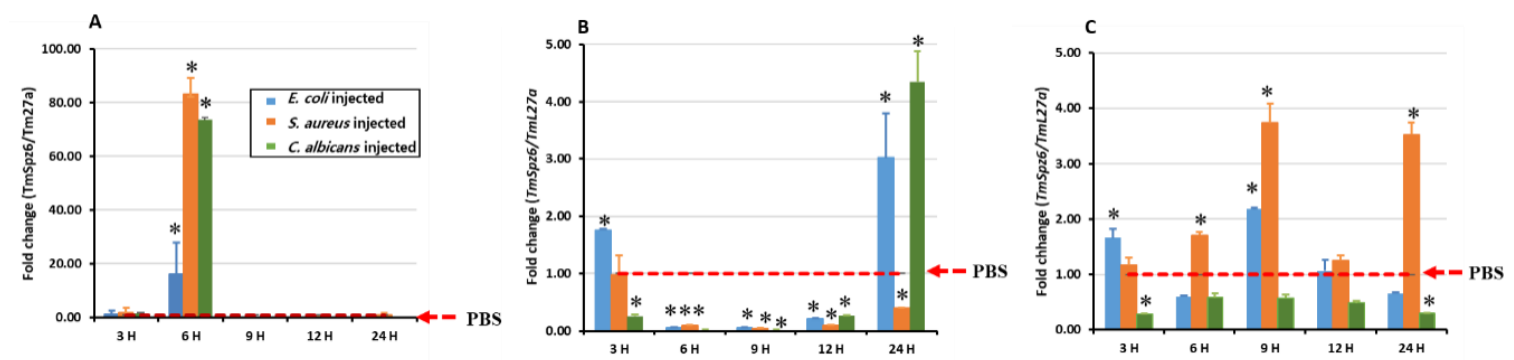

Figure 4. Induction patterns of TmSpz6 expression in different T. molitor larval tissues. The temporal expression of TmSpz6 was analyzed in the hemocytes (A), gut (B), and fat body (C) of young larvae at $3,6,9,12$, and $24 \mathrm{~h}$ post-injection of $E$. coli $\left(10^{6}\right.$ cells $\left./ \mu \mathrm{L}\right), S$. aureus $\left(10^{6}\right.$ cells $\left./ \mu \mathrm{L}\right)$, and $C$. albicans $(5$ $\times 10^{4}$ cells $/ \mu \mathrm{L}$ ). Twenty young larvae were used at each time point. TmSpz 6 expression levels were normalized to those in $1 \mathrm{X}$ phosphate-buffered saline (1X PBS)-injected controls. T. molitor 60S ribosomal protein L27a (TmL27a) was used as an internal control. The asterisks indicate significant differences $(p \leq 0.05)$.

\subsection{Effect of TmSpz6 Silencing on T. molitor Survival}

Since the temporal induction of TmSpz6 was observed following microorganism injection, the survival of TmSpz6-silenced T. molitor larvae in response to infection was assessed. The survival of TmSpz6-silenced or dsEGFP-injected T. molitor larvae after microbial injection was monitored for 10 days. The results show that the injection of dsTmSpz 6 or $\mathrm{d} E G F P$ did not affect the survival of T. molitor larvae after injection with PBS. However, dsTmSpz6-injected larvae were highly susceptible to $E$. coli $(68.5 \%)$ and S. aureus (57.5\%) (Figure 5B,C), whereas their survival rate after infection with C. albicans was not significantly different from that of the controls (Figure 5D).

\subsection{Effects of TmSpz6 Gene Silencing on the Expression of AMP Genes}

Based on the results of the survival study, the importance of TmSpz6 in the immune defense against gram-negative and gram-positive bacteria was postulated. Thus, the function of TmSpz6 in the production of AMPs in response to microbial infection was investigated by silencing the expression of TmSpz6 in T. molitor larvae, challenging them with E. coli, S. aureus, or C. albicans, and assessing the expression levels of 14 different AMP genes at $24 \mathrm{~h}$ post-infection.

The results showed that TmSpz6 silencing significantly downregulated mainly the expression of TmTen-2 in hemocytes (Figure 6A) and gut (Figure 6B). In addition, the expression of TmTen-3 in fat body was significantly downregulated (Figure 6C). Similarly, TmCec-2 in hemocytes (Figure S1), Tene-4, TmTLP-1, and TmCec-2 in the fat body (Figure S2); and TmTen-1, -2, -4, TmCol-1, TmTLP-1, TmTLP-2, and TmCec-2 in the gut (Figure S3) were significantly downregulated following the injection with $E$. coli. Similarly, TmCec-2 in hemocytes (Figure S1); TmTen-2, -3, TmAtt-1a, TmAtt-1b, TmDef-1, TmDef-2, TmTLP-2, and TmCec-2 in the fat body (Figure S2); and TmTen-1,-2,-3,-4, TmAtt-1a, TmAtt-1b, TmCol-1, TmCol-2, TmDef-2, and TmCec-2 in the gut (Figure S3) were considerably suppressed in TmSpz6-silenced larvae injected with $S$. aureus.

Following C. albicans injection, TmTen-1, -4, TmAtt-1a, -1b,-2, TmCol-1, TmDef-1, -2, and TmCol-2 were significantly downregulated in the hemocytes of TmSpz6-silenced larvae (Figure S1). Additionally, TmDef-2, TmTLP-2, and TmCec-2 in the fat body (Figure S2), and TmTLP-1, and TmTLP-2 in the gut (Figure S3) were markedly downregulated in TmSpz6-silenced larvae. Interestingly, in contrast, TmSpz6 knockdown increased the mRNA levels of TmTen-1, -3, TmAtt-1a,-1b,-2, TmCol-1,-2, and TmDef-1 in the hemocytes of E. coli-challenged larvae. 

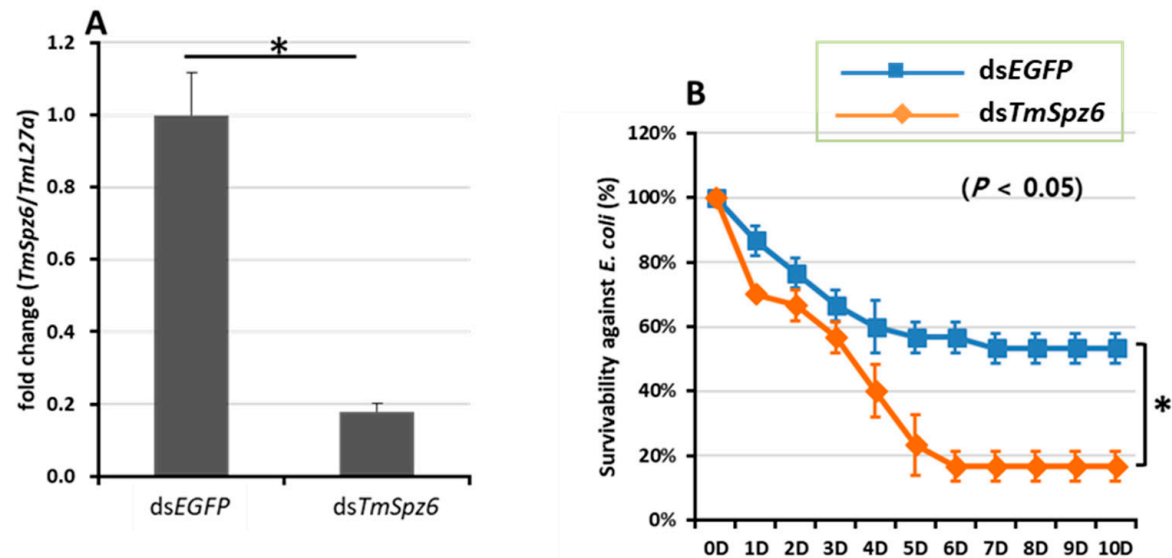

C
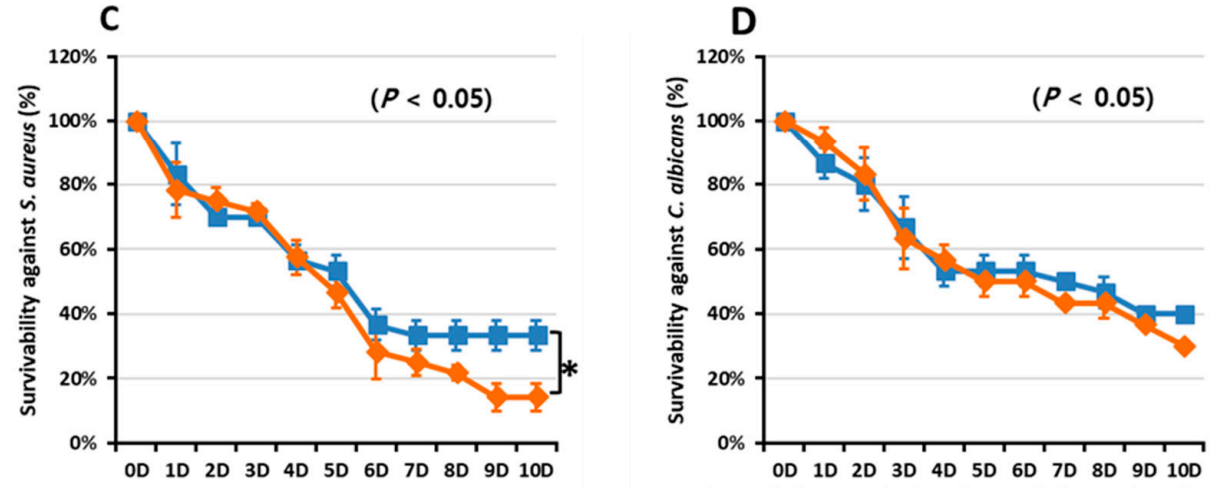

Time $(\mathrm{H})$ post injection of the microbes

Figure 5. Effect of dsTmSpz6 on the survival of T. molitor larvae. Silencing efficiency of TmSpz6 mRNA was measured by qRT-PCR at 3 days post-injection (A). Then, the TmSpz6-silenced larvae were injected with E. coli (B), S. aureus (C), and C. albicans (D). dsEGFP-injected larvae were used as a negative control. The data are an average of three independent biological replicate experiments. The asterisks indicate significant differences between dsTmSpz6- and dsEGFP-treated groups $(p<0.05)$.
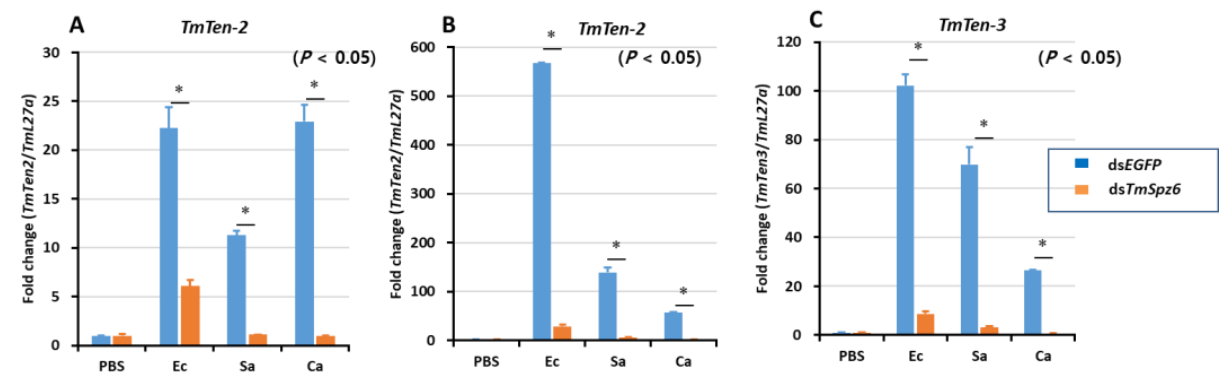

Figure 6. The antimicrobial peptide expression levels in TmSpz6-knockdown T. molitor larval were performed by injecting either E. coli (Ec), S. aureus (Sa), or C. albicans (Ca) on the 3th day post-TmSpz6 silencing. $24 \mathrm{~h}$ post microbial challenge, the known immune tissues including hemocytes (A), gut (B), and fat body (C) were dissected. Then the expression levels of AMPs including TmTenecin-1 (TmTene-1), TmTenecin-2(TmTene-2), TmTenecin-3 (TmTene-3), TmTenecin-4 (TmTene-4), TmAttacin-1a (TmAtt-1a), TmAttacin-1b (TmAtt-1b), TmAttacin-2 (TmAtt-2), TmDefensin-1 (TmDef-1), TmDefensin-2 (TmDef-2), TmColeptericin-1 (TmCole-1), TmColeptericin-2 (TmCole-2), TmCecropin-2 (TmCec-2), TmTLP-1 (TmTLP-1) and TmTLP2 (TmTLP-2) were measured by qRT-PCR. dsEGFP was used as a negative control and TmL27a was used as an internal control. All experiments were triplicated. The asterisks represent significant differences between dsTmSpz6-and dsEGFP-treated groups when compared by the Student's $t$-test $(p \leq 0.05)$. 


\section{Discussion}

AMPs are essential effectors of the innate immune defense system against numerous pathogens $[29,30]$. In insects, the expression of AMPs is mainly regulated by the evolutionarily conserved Toll and IMD pathways [12]. Specifically, the Toll pathway, a well-known activator of AMP production in insects, is activated by the extracellular ligand spätzle [9]. The functions of the spätzle proteins in the innate immunity of various insect species have been repeatedly reported. In Drosophila, the most widely studied insect species, the functions of spätzle proteins in the defense response against microbial infections have been well studied [5,17,31]. The importance of spätzle in the activation of the Toll receptor and the subsequent activation of AMP production has also been studied in the lepidopteran insects, B. mori [32], and M. sexta [33].

In this study, TmSpz6 was identified in T. molitor and functionally characterized. TmSpz6 mRNA was highly expressed in the prepupal and pupal stages, whereas comparatively lower expression levels were observed in the larval and adult stages. Tissue-specific expression studies showed that TmSpz6 was expressed at various levels in all examined tissues in the larval and adult stages. The observed variations in TmSpz6 expression in different tissues and developmental stages is assumed to be controlled by developmental hormones. This assumption is based on previous studies demonstrating the importance of juvenile hormone $(\mathrm{JH})$ and ecdysone in the insect innate immune response. For example, ecdysone was shown to activate PGRP-LC expression and induce AMP expression in Drosophila [34], JH was shown to act as an AMP-activator in B. mori [35], and ecdysone enhanced the B. mori immune system [36]. Therefore, it is possible that there are similar hormonal effects on TmSpz6 expression in T. molitor, caused by either ecdysone or other developmental hormones. Thus, the fluctuations in the expression of TmSpz6 at the same developmental stage or different stage are assumed to be due to hormonal changes. Briefly, the expression of TmSpz6 was highest at the perpupal stage and in 3- and 6-day-old pupae. These are transitional stages that the ecdysone commonly controls. The investigation of the expression of ecdysone during the development of Drosophila by radioimmune assay showed that ecdysone activity was highest during the pupal, prepupal, and late larval stages (in descending order) [37] In addition, TmSpz6 expression was highest in the hemocytes of larvae and adults. Previously, it has been reported that hemocytes play important roles in immunity, nutrient transportation, and growth hormone synthesis [38,39]. Furthermore, hemocytes are known to express spätzle proteins [40] and our results confirm this since we found the highest levels of TmSpz6 expression in hemocytes, suggesting that this tissue is important in T. molitor immune response. Taken together, these data suggest that TmSpz6 expression during transitional stages and in growth hormone-synthesizing tissues (hemocytes) under normal conditions is regulated by developmental hormones.

The active form of spätzle is generated during development [41] and/or the immune response to microbial infection [12,42]. Specifically, PGRP-SA or GNBP1 recognizes conserved bacterial or fungal molecules and activates the serine protease cascade that leads to the generation of mature spätzle [8,9]. The Toll receptor for the spätzle ligand is known to be located on the plasma membrane of fat body and hemocyte cells [43-45]. Therefore, to examine the induction of the TmSpz6-related immune response in T. molitor, larvae were challenged with E. coli, S. aureus, or C. albicans and then the expression of TmSpz6 was assessed in the main immune organs, such as hemocytes, fat body, and the gut. TmSpz6 was found to be highly induced at different time points following the injection of all tested microorganisms. Spätzle genes are activated upon microorganism infection as an effector of the Toll pathway. In $M$. sexta, spätzle-1 was upregulated at $24 \mathrm{~h}$ post-injection of E. coli or insoluble $\beta$-1,3-glucan [33]. In the current study, the highest TmSpz6 expression was observed in hemocytes against the three pathogens. The well-known gram-negative bacteria, E. coli possesses DAP-type PGN, which is recognized by PGRP-LC or -LE to activate the IMD pathway in Drosophila [46]. However, it is not surprising that a gram-negative bacterium (E. coli) induced the expression of TmSpz6 in current study, as it has already been reported that Tenebrio PGRP-SA can recognize the polymeric DAP-type peptidoglycan present in E. coli to activate the Toll receptor ligand spätzle in T. molitor [47]. 
The importance of TmSpz6 in the immune response against microbial infection was studied using RNAi. TmSpz6-silenced larvae showed significant susceptibility starting from 4th day of E. coli injection. This indicates that TmSpz6 plays a key role in the microbial defense response in T. molitor larvae. The expression of insect AMPs is mainly regulated by the evolutionarily conserved Toll and IMD pathways. Particularly, the spätzle-Toll-MyD88-Tube-Pell complexes and intracellular Dorsal and Dif binding to $\mathrm{kB}$-related sequences play key roles in AMP production [12]. Spätzle plays a paramount role in activating Toll and is itself activated by other complexes [9]. Additionally, AMP gene expression was examined in TmSpz6-silenced larvae to investigate its function in the expression of AMP genes in response to microbial infection. The results reveal that TmSpz6 silencing suppressed the induction of several AMP gene expressions in response to microbial infection, supporting the survival study results. Most of the AMP genes that were suppressed when TmSpz6 was silenced encode AMPs that have activity against gram-negative bacteria, such as E. coli (Tene-2, Cec-2, Tene-4, and Cole-1) [24,47-50], and gram-positive bacteria, such as S. aureus (Tene-3, Def-1, Def-2, Cec-2 and Tene-1) [51-54].

\section{Conclusions}

This study focused on the identification and immunological functional characterization of TmSpz6 in T. molitor. TmSpz6 is highly expressed in prepupal and 3-day-old pupal stages. In the tissue specific expression study, TmSpz 6 was highly expressed in the hemocytes of late larvae. The induction patterns in response to microbial challenges revealed that TmSpz 6 was highly upregulated in hemocytes at the 6 h-post injection of E. coli, S. aureus, and C. albicans. The survivability of TmSpz6-silenced larvae significantly decreased after the injection of E. coli or S. aureus. The loss of function of TmSpz6 significantly reduced the induction of various AMPs, particularly TmTen-2 and -3 in response to E. coli and S. aureus infection. Taken together, these results suggest that TmSpz6 plays an important role in regulating AMP expression and increases the survival of T. molitor against E. coli and S. aureus.

Supplementary Materials: The following are available online at http://www.mdpi.com/2075-4450/11/2/105/s1, Figure S1: Antimicrobial peptide (AMP) gene induction patterns in the hemocytes of TmSpz6-silenced larvae. Figure S2: Antimicrobial peptide (AMP) gene induction patterns in the fat body of TmSpz6-silenced larvae. Figure S3: Antimicrobial peptide (AMP) gene induction patterns in the gut of TmSpz6-silenced larvae.

Author Contributions: Y.S.H. and Y.H.J. conceived and designed the experiments; Y.S.H., and Y.H.J. contributed reagents/materials/analysis tools; T.T.E., M.K., Y.M.B. and D.H.K. performed the experiments; T.T.E. analyzed the data; T.T.E., wrote first draft of the manuscript; Y.H.J. and Y.S.H. and Y.S.L. revised the manuscript. All authors have read and agreed to the published version of the manuscript.

Funding: This research was supported by Basic Science Research Program through the National Research Foundation of Korea (NRF) funded by the Ministry of Science, ICT, and future Planning (Grant No. 2018R1A2A2A05023367).

Conflicts of Interest: The authors declare no conflict of interest.

\section{References}

1. Jiang, Y.-y. A review of researches on agglutinins of Lepidoptera insect. Entomol. J. East China 2006, 15, 25-29.

2. Imler, J.-L.; Bulet, P. Antimicrobial Peptides in Drosophila: Structures, Activities and Gene Regulation, in Mechanisms of Epithelial Defense; Karger Publishers: Basel, Switzerland, 2005; pp. 1-21.

3. Wang, X.; Zhang, Y.; Zhang, R.; Zhang, J. The diversity of pattern recognition receptors (PRRs) involved with insect defence against pathogens. Curr. Opin. Insect Sci. 2019. [CrossRef]

4. Levitin, A.; Whiteway, M. Drosophila innate immunity and response to fungal infections. Cell. Microbiol. 2008, 10, 1021-1026. [CrossRef]

5. Ferrandon, D.; Imler, J.-L.; Hetru, C.; Hoffmann, J.A. The Drosophila systemic immune response: Sensing and signalling during bacterial and fungal infections. Nat. Rev. Immunol. 2007, 7, 862. [CrossRef]

6. De Gregorio, E.; Spellman, P.T.; Tzou, P.; Rubin, G.M.; Lemaitre, B. The Toll and Imd pathways are the major regulators of the immune response in Drosophila. EMBO J. 2002, 21, 2568-2579. [CrossRef] [PubMed] 
7. Lemaitre, B.; Nicolas, E.; Michaut, L.; Reichhart, J.-M.; Hoffmann, J.A. The dorsoventral regulatory gene cassette spätzle/Toll/cactus controls the potent antifungal response in Drosophila adults. Cell 1996, 86, 973-983. [CrossRef]

8. El Chamy, L.; Leclerc, V.; Caldelari, I.; Reichhart, J.-M. Sensing of 'danger signals' and pathogen-associated molecular patterns defines binary signaling pathways 'upstream' of Toll. Nat. Immunol. 2008, 9, 1165. [CrossRef] [PubMed]

9. Roh, K.-B.; Kim, C.-H.; Lee, H.; Kwon, H.-M.; Park, J.-W.; Ryu, J.-H.; Kurokawa, K.; Ha, N.-C.; Lee, W.-J.; Lemaitre, B. Proteolytic cascade for the activation of the insect toll pathway induced by the fungal cell wall component. J. Biol. Chem. 2009, 284, 19474-19481. [CrossRef] [PubMed]

10. Gobert, V.; Gottar, M.; Matskevich, A.A.; Rutschmann, S.; Royet, J.; Belvin, M.; Hoffmann, J.A. Dual activation of the Drosophila toll pathway by two pattern recognition receptors. Science 2003, 302, 2126-2130. [CrossRef]

11. Anderson, K.V. Toll signaling pathways in the innate immune response. Curr. Opin. Immunol. 2000, 12, 13-19. [CrossRef]

12. Valanne, S.; Wang, J.-H.; Rämet, M. The Drosophila toll signaling pathway. J. Immunol. 2011, 186, 649-656. [CrossRef] [PubMed]

13. DeLotto, Y.; Smith, C.; DeLotto, R. Multiple isoforms of the Drosophila Spätzle protein are encoded by alternatively spliced maternal mRNAs in the precellular blastoderm embryo. Mol. Gen. Genet. 2001, 264, 643-652. [CrossRef] [PubMed]

14. Arnot, C.J.; Gay, N.J.; Gangloff, M. Molecular mechanism that induces activation of Spätzle, the ligand for the Drosophila Toll receptor. J. Biol. Chem. 2010, 285, 19502-19509. [CrossRef]

15. Kim, C.-H.; Kim, S.-J.; Kan, H.; Kwon, H.-M.; Roh, K.-B.; Jiang, R.; Yang, Y.; Park, J.-W.; Lee, H.-H.; Ha, N.-C. A three-step proteolytic cascade mediates the activation of the peptidoglycan-induced toll pathway in an insect. J. Biol. Chem. 2008, 283, 7599-7607. [CrossRef] [PubMed]

16. Weber, A.N.; Tauszig-Delamasure, S.; Hoffmann, J.A.; Lelièvre, E.; Gascan, H.; Ray, K.P.; Morse, M.A.; Imler, J.-L.; Gay, N.J. Binding of the Drosophila cytokine Spätzle to Toll is direct and establishes signaling. Nat. Immunol. 2003, 4, 794. [CrossRef] [PubMed]

17. Jang, I.-H.; Chosa, N.; Kim, S.-H.; Nam, H.-J.; Lemaitre, B.; Ochiai, M.; Kambris, Z.; Brun, S.; Hashimoto, C.; Ashida, M. A Spätzle-processing enzyme required for toll signaling activation in Drosophila innate immunity. Dev. Cell 2006, 10, 45-55. [CrossRef] [PubMed]

18. Wang, Y.; Cheng, T.; Rayaprolu, S.; Zou, Z.; Xia, Q.; Xiang, Z.; Jiang, H. Proteolytic activation of pro-spätzle is required for the induced transcription of antimicrobial peptide genes in lepidopteran insects. Dev. Comp. Immunol. 2007, 31, 1002-1012. [CrossRef] [PubMed]

19. Zhong, X.; Xu, X.-X.; Yi, H.-Y.; Lin, C.; Yu, X.-Q. Toll-Spätzle pathway in the tobacco hornworm, Manduca sexta. Insect Biochem. Mol. Biol. 2012, 42, 514-524. [CrossRef]

20. Luna, C.; Wang, X.; Huang, Y.; Zhang, J.; Zheng, L. Characterization of four Toll related genes during development and immune responses in Anopheles gambiae. Insect Biochem. Mol. Biol. 2002, 32, 1171-1179. [CrossRef]

21. Shin, S.W.; Bian, G.; Raikhel, A.S. A toll receptor and a cytokine, Toll5A and Spz1C, are involved in toll antifungal immune signaling in the mosquito Aedes aegypti. J. Biol. Chem. 2006, 281, 39388-39395. [CrossRef]

22. Shi, X.-Z.; Zhang, R.-R.; Jia, Y.-P.; Zhao, X.-F.; Yu, X.-Q.; Wang, J.-X. Identification and molecular characterization of a Spätzle-like protein from Chinese shrimp (Fenneropenaeus chinensis). Fish Shellfish. Immunol. 2009, 27, 610-617. [CrossRef] [PubMed]

23. Wang, P.-H.; Liang, J.-P.; Gu, Z.-H.; Wan, D.-H.; Weng, S.-P.; Yu, X.-Q.; He, J.-G. Molecular cloning, characterization and expression analysis of two novel Tolls (LvToll2 and LvToll3) and three putative Spätzle-like Toll ligands (LvSpz1-3) from Litopenaeus vannamei. Dev. Comp. Immunol. 2012, 36, 359-371. [CrossRef] [PubMed]

24. Chae, J.-H.; Kurokawa, K.; So, Y.-I.; Hwang, H.O.; Kim, M.-S.; Park, J.-W.; Jo, Y.-H.; Lee, Y.S.; Lee, B.L. Purification and characterization of tenecin 4, a new anti-Gram-negative bacterial peptide, from the beetle Tenebrio molitor. Dev. Comp. Immunol. 2012, 36, 540-546. [CrossRef] [PubMed]

25. Keshavarz, M.; Jo, Y.H.; Park, K.B.; Ko, H.J.; Edosa, T.T.; Lee, Y.S.; Han, Y.S. Tm DorX2 positively regulates antimicrobial peptides in Tenebrio molitor gut, fat body, and hemocytes in response to bacterial and fungal infection. Sci. Rep. 2019, 9, 1-19. [CrossRef] 
26. Park, S.; Jo, Y.H.; Park, K.B.; Ko, H.J.; Kim, C.E.; Bae, Y.M.; Kim, B.; Jun, S.A.; Bang, I.S.; Lee, Y.S. TmToll-7 plays a crucial role in innate immune responses against Gram-negative bacteria by regulating 5 AMP genes in Tenebrio molitor. Front. Immunol. 2019, 10, 310. [CrossRef]

27. Yaffe, H.; Buxdorf, K.; Shapira, I.; Ein-Gedi, S.; Moyal-Ben Zvi, M.; Fridman, E.; Moshelion, M.; Levy, M. LogSpin: A simple, economical and fast method for RNA isolation from infected or healthy plants and other eukaryotic tissues. BMC Res. Notes 2012, 5, 45. [CrossRef]

28. Livak, K.J.; Schmittgen, T.D. Analysis of relative gene expression data using real-time quantitative PCR and the 2- $\Delta \Delta C$ T method. Methods 2001, 25, 402-408. [CrossRef]

29. Yi, H.-Y.; Chowdhury, M.; Huang, Y.-D.; Yu, X.-Q. Insect antimicrobial peptides and their applications. Appl. Microbiol. Biotechnol. 2014, 98, 5807-5822. [CrossRef]

30. Wu, Q.; Patočka, J.; Kuča, K. Insect antimicrobial peptides, a mini review. Toxins 2018, 10, 461. [CrossRef]

31. Tanji, T.; Xiaodi, H.; Alexander, N.R.; Weber, Y.; Tony, I. Toll and IMD pathways synergistically activate an innate immune response in Drosophila melanogaster. Mol. Cell. Biol. 2007, 27, 4578-4588. [CrossRef]

32. Huang, X.; Xu, Y.; Zhang, Y.; Liu, J.; Luo, S.; Wu, J. Spatzle4 gene of silkworm, Bombyx mori: Identification, immune response, and the effect of RNA interference on the antimicrobial peptides' expression in the integument. Saudi J. Biol. Sci. 2018, 25, 1817-1825. [CrossRef]

33. An, C.; Jiang, H.; Kanost, M.R. Proteolytic activation and function of the cytokine Spatzle in the innate immune response of a lepidopteran insect, Manduca sexta. FEBS J. 2010, 277, 148-162. [CrossRef]

34. Rus, F.; Flatt, T.; Tong, M.; Aggarwal, K.; Okuda, K.; Kleino, A.; Yates, E.; Tatar, M.; Silverman, N. Ecdysone triggered PGRP-LC expression controls Drosophila innate immunity. EMBO J. 2013, 32, 1626-1638. [CrossRef]

35. Tian, L.; Guo, E.; Diao, Y.; Zhou, S.; Peng, Q.; Cao, Y.; Ling, E.; Li, S. Genome-wide regulation of innate immunity by juvenile hormone and 20-hydroxyecdysone in the Bombyx fat body. BMC Genom. 2010, 11, 549. [CrossRef]

36. Sun, W.; Shen, S.-H.; Zhou, L.-X.; Zhang, Z. ecdysone titer determined by 3DE-3 $\beta$-reductase enhances the immune response in the silkworm. J. Immunol. 2016, 196, 1646-1654. [CrossRef]

37. Borst, D.W.B.; Walter, E.; O'Connor, J.D.; King, D.S.; Fristrom, J.W. Ecdysone levels during metamorphosis of Drosophila melanogaster. Dev. Biol. 1974, 39, 308-316. [CrossRef]

38. Ling, E.; Shirai, K.; Kanekatsu, R.; Kiguchi, K. Hemocyte differentiation in the hematopoietic organs of the silkworm, Bombyx mori: Prohemocytes have the function of phagocytosis. Cell Tissue Res. 2005, 320, 535-543. [CrossRef]

39. Merchant, D.; Ertl, R.L.; Rennard, S.I.; Stanley, D.W.; Miller, J.S. Eicosanoids mediate insect hemocyte migration. J. Insect Physiol. 2008, 54, 215-221. [CrossRef]

40. Shia, A.K.; Glittenberg, M.; Thompson, G.; Weber, A.N.; Reichhart, J.-M.; Ligoxygakis, P. Toll-dependent antimicrobial responses in Drosophila larval fat body require Spätzle secreted by haemocytes. J. Cell Sci. 2009, 122, 4505-4515. [CrossRef]

41. Ursel, C.; Fandrich, U.; Hoffmann, A.; Sieg, T.; Ihling, C.; Stubbs, M.T. In vitro maturation of Drosophila melanogaster Spätzle protein with refolded Easter reveals a novel cleavage site within the prodomain. Biol. Chem. 2013, 394, 1069-1075. [CrossRef]

42. Stokes, B.A.; Yadav, S.; Shokal, U.; Smith, L.C.; Eleftherianos, I. Bacterial and fungal pattern recognition receptors in homologous innate signaling pathways of insects and mammals. Front. Microbiol. 2015, 6, 19. [CrossRef]

43. Rämet, M.; Manfruelli, P.; Pearson, A.; Mathey-Prevot, B.; Ezekowitz, R.A.B. Functional genomic analysis of phagocytosis and identification of a Drosophila receptor for E. coli. Nature 2002, 416, 644. [CrossRef]

44. Rämet, M.; Pearson, A.; Manfruelli, P.; Li, X.; Koziel, H.; Göbel, V.; Chung, E.; Krieger, M.; Ezekowitz, R.A.B. Drosophila scavenger receptor $\mathrm{CI}$ is a pattern recognition receptor for bacteria. Immunity 2001, 15, 1027-1038. [CrossRef]

45. Tsakas, S.; Marmaras, V. Insect immunity and its signalling: An overview. Invertebr. Surviv. J. 2010, 7, 228-238.

46. Kaneko, T.; Goldman, W.E.; Mellroth, P.; Steiner, H.; Fukase, K.; Kusumoto, S.; Harley, W.; Fox, A.; Golenbock, D.; Silverman, N. Monomeric and polymeric gram-negative peptidoglycan but not purified LPS stimulate the Drosophila IMD pathway. Immunity 2004, 20, 637-649. [CrossRef]

47. Yu, Y. Diversity of innate immune recognition mechanism for bacterial polymeric meso-diaminopimelic acid-type peptidoglycan in insects. J. Biol. Chem. 2010, 285, 32937-32945. [CrossRef] 
48. Bulet, P.; Cociancich, S.; Dimarcq, J.-L.; Lambert, J.; Reichhart, J.-M.; Hoffmann, D.; Hetru, C.; Hoffmann, J.A. Insect immunity. Isolation from a coleopteran insect of a novel inducible antibacterial peptide and of new members of the insect defensin family. J. Biol. Chem. 1991, 266, 24520-24525.

49. Carlsson, A.; Engström, P.; Palva, E.T.; Bennich, H. Attacin, an antibacterial protein from Hyalophora cecropia, inhibits synthesis of outer membrane proteins in Escherichia coli by interfering with omp gene transcription. Infect. Immun. 1991, 59, 3040-3045. [CrossRef]

50. Jo, Y.H.; Park, S.; Park, K.B.; Noh, M.Y.; Cho, J.H.; Ko, H.J.; Kim, C.E.; Patnaik, B.B.; Kim, J.; Won, R.; et al. In silico identification, characterization and expression analysis of attacin gene family in response to bacterial and fungal pathogens in Tenebrio molitor. Entomol. Res. 2018, 48, 45-54. [CrossRef]

51. Moon, H.J.; Lee, S.Y.; Kurata, S.; Natori, S.; Lee, B.L. Purification and Molecular Cloning of cDNA for an Inducible Antibacterial Protein from Larvae of the Coleopteran, Tenebrio molitor1. J. Biochem. 1994, 116, 53-58. [CrossRef]

52. Park, S.-I.; Kim, J.-W.; Yoe, S.M. Purification and characterization of a novel antibacterial peptide from black soldier fly (Hermetia illucens) larvae. Dev. Comp. Immunol. 2015, 52, 98-106. [CrossRef]

53. Li, Z.; Mao, R.; Teng, D.; Hao, Y.; Chen, H.; Wang, X.; Wang, X.; Yang, N.; Wang, J. Acterial and immunomodulatory activities of insect defensins-DLP2 and DLP4 against multidrug-resistant Staphylococcus aureus. Sci. Rep. 2017, 7, 12124. [CrossRef]

54. Bulet, P.; Stocklin, R. Insect antimicrobial peptides: Structures, properties and gene regulation. Protein Pept. Lett. 2005, 12, 3-11. [CrossRef]

(C) 2020 by the authors. Licensee MDPI, Basel, Switzerland. This article is an open access article distributed under the terms and conditions of the Creative Commons Attribution (CC BY) license (http://creativecommons.org/licenses/by/4.0/). 